\title{
Fatal Gestational Trophoblastic Neoplasia
} John R Lurain*

Department of Obstetrics and Gynecology, Feinberg School of Medicine, Northwestern University, USA

\section{Introduction}

Gestational trophoblastic neoplasia (GTN) includes invasive mole, choriocarcinoma, placental site trophoblastic tumor (PSTT) and epithelioid trophoblastic tumor (ETT). The overall cure rate in treating these tumors is currently $>90 \%$ : virtually $100 \%$ for nonmetastatic disease and $80-90 \%$ for metastatic disease. This success is the result of: (1) the inherent sensitivity of trophoblastic neoplasms to chemotherapy; (2) the effective use of the tumor marker human chorionic gonadotropin (hCG) for diagnosis and monitoring therapy; (3) the referral of patients to or consultation with clinicians who have special expertise in management of these relatively rare diseases; (4) the identification of prognostic factors that predicts treatment response and enhances individualization of therapy; (5) the use of combined modality treatment with multiagent chemotherapy, radiation and surgery in the highest risk patients [1].

Risk factors related to treatment failure for GTN that have been identified include histopathologic diagnosis of choriocarcinoma, high initial hCG level, long interval from preceding pregnancy event to diagnosis, antecedent non-molar pregnancy, multiple sites and increasing number of metastases and extent of prior treatment [2]. Understanding the causes of fatal GTN, including delayed diagnosis, failed treatments, sites of resistant disease and psychosocial factors, is important for preventing future deaths from GTN.

In 2015 we published an analysis of GTN treatment failures at the Brewer Trophoblastic Disease Center of Northwestern University to determine clinical factors that contributed to death comparing 19792012 to 1962-1978 [3-5]. Death from GTN occurred in 19 (4\%) of 483 patients treated from 1979-2012 compared to 45 (11\%) of 396 patients treated from 1962-1978 $(\mathrm{p}<0.001)$. Pretreatment hCG levels, time from pregnancy event to treatment, non-molar antecedent pregnancy rate and use of surgery to control metastatic disease were similar between the two treatment eras. Patients in the more recent series were more likely to have presented with FIGO stage IV disease +/- brain metastasis, more likely to have been initially treated with multiagent chemotherapy and less likely to have received treatment before referral to the Brewer Center compared to the previous group. Patients treated from 1979-2012 more often died from chemotherapy-resistant disease leading to multiorgan failure or pulmonary insufficiency ( $79 \%$ vs. $40 \%$, $\mathrm{p}<0.01$ ), while patients treated from 1962-1978 more frequently died from hemorrhage ( $42 \%$ vs. $11 \%$, p $<0.02)$. Only $2(11 \%)$ of the patients from our most recent series died within one month of diagnosis compared to 11 (24\%) of the patients treated before 1979. Median time from diagnosis to death also increased from 6 months to 22 months.

Our findings were consistent with other published experiences of fatal GTN. Case series from China and the United Kingdom noted death rates of $5.6 \%$ and $2.7 \%$, respectively, similar to our most recent series where the death rate was 3.9\% [6,7]. In both the Chinese and United Kingdom studies, the main causes of death were multiorgan failure, intracranial hemorrhage and/or respiratory failure. However, in the Chinese study, many more patients died during the acute phase of their disease, possibly reflecting social factors and barriers to healthcare resulting in later presentations with more advanced disease. Whereas, in the United Kingdom, all women with gestational trophoblastic disease are registered and treated at one of two centers, presumably resulting in earlier disease presentation, consistent treatment and improved results. As in our series, the Dutch reported a major decline in the number of deaths from GTN after 1980, presumably due to the introduction of etoposide into the treatment regimen for high-risk patients, as well as a reduction in early deaths due to hemorrhage with most patients in later years dying of widely metastatic disease [8]. The French reported a 5 year overall mortality rate of $2 \%$ for patients with GTN, excluding PSTT/ETT. High-risk patients with a FIGO score $>12$ had a mortality rate of $12 \%$ and accounted for $52 \%$ of the deaths in the entire cohort, including 6 of the 8 patients who died within four weeks of treatment initiation [9]. In a worldwide survey, Kohorn identified better survival for patients who received initial treatment at a trophoblastic disease center. The mortality rate was $2.1 \%$ for patients treated primarily at a trophoblast center compared to $8 \%$ for patients referred to a center after failure of primary treatment elsewhere [10].

At our center, the overall survival rate in patients with GTN improved from $89 \%$ in $1962-1978$ to $96 \%$ in $1979-2012$. The most common cause of death also changed from hemorrhage from metastatic sites in our earlier series to widespread chemotherapyresistant disease in our later series. Factors primarily responsible for current treatment failure included inadequate initial therapy in $32 \%$ of patients, the presence of extensive disease at the time of presentation with all patients having FIGO stages III or IV: scores 9-19 (mean=14), and psychosocial factors that precluded adequate therapy in $21 \%$ of patients.

The challenge remains to provide all patients with GTN appropriate therapy for the greatest chance of cure. Timely request for advice from or referral for treatment to clinicians with expertise in management of GTN is recommended for a patient who fails initial therapy for lowrisk disease (FIGO score $<7$ ) and for any patient with high-risk disease (FIGO stage IV and/or score $\geq 7$ ).

\section{References}

1. Lurain JR (2011) Gestational trophoblastic disease. II classification and management of gestational trophoblastic neoplasia. Am J Obstet Gynecol 204: $11-18$.

2. Lurain JR, Casanova LA, Miller DS, Rademaker AW (1991) Prognostic factors in gestational trophoblastic tumors. Am J Obstet Gynecol 164: 611-616.

3. Lurain JR (1987) Causes of treatment failure in gestational trophoblastic disease. J Reprod Med 32: 675-679.

*Corresponding author: John R Lurain, Department of Obstetrics and Gynecology, Feinberg School of Medicine, Northwestern University, USA, Tel: 312-472-4684; Fax: 312-472-4688; E-mail: jlurain@nm.org

Received September 03, 2016; Accepted October 26, 2016; Published November 02, 2016

Citation: Lurain JR (2016) Fatal Gestational Trophoblastic Neoplasia. J Oncol Transl Res 2: 111. doi: 10.4172/2476-2261.1000111

Copyright: (c) 2016 Lurain JR. This is an open-access article distributed under the terms of the Creative Commons Attribution License, which permits unrestricted use, distribution, and reproduction in any medium, provided the original author and source are credited. 
4. Neubauer NL, Strohl AE, Schink JC, Lurain JR (2015) Fatal gestational trophoblastic neoplasia: An analysis of treatment failures at the Brewer Trophoblastic Disease Center from 1979-2012 compared to 1962-1978. Gynecol Oncol 138: 339-342.

5. Lurain JR, Brewer JI, Mazur MT, Torok EE (1982) Fatal gestational trophoblastic disease: An analysis of treatment failures. Am J Obstet Gynecol 144: 391-395.

6. Yang JX, Wan Y, Li XR (2006) Analysis of death causes of gestationa trophoblastic neoplasia patients. Zhonghua Fu Chan Ke Za Zhi 41: 403-407.

7. Kingdon SJ, Coleman RE, Ellis L, Hancock BW (2012) Deaths from gestational trophoblastic neoplasia. Any lessons to be learned? J Reprod Med 57: 293-296

8. Lybol C, Centen DW, Thomas CMG (2012) Fatal cases of gestational trophoblastic neoplasia over four decades in the Netherlands: A retrospective cohort study. BJOG 119: 1465-1472.

9. Bolze PA, Riedl C, Massardier J (2016) Mortality rate of gestational trophoblastic neoplasia with a FIGO score $\geq 13$. Am J Obstet Gynecol 214: 390-391.

10. Kohorn El (2014) Worldwide survey of the results of treating gestational trophoblastic disease. J Reprod Med 59: 145-153. 